Article

\title{
Classical Mechanics as a Fundamental Law of Quantum Mechanics
}

\author{
Yehuda Roth ${ }^{1}$ (1)* \\ 1 Oranim College; yudroth@gmail.com
}

\begin{abstract}
In our previous paper, we showed that the so-called quantum entanglement also exists in classical mechanics. The inability to measure this classical entanglement was rationalized with the definition of a classical observer which collapses all entanglement into distinguishable states. It was shown that evidence for this primary coherence is Newton's third law. However, in reformulating a "classical entanglement theory" we assumed the existence of Newton's second law as an operator form where a force operator was introduced through a Hilbert space of force states. In this paper, we derive all related physical quantities and laws from basic quantum principles. We not only define a force operator but also derive the classical mechanic's laws and prove the necessity of entanglement to obtain Newton's third law.
\end{abstract}

Keywords: post collapse entanglement; commutators pictures; force operator; labeling set

\section{Introduction}

Quantum resource theories, quantum entanglement in particular [3], are considered to be beyond classical understanding, offering a highly versatile and powerful framework for studying different phenomena in quantum physics [4]. In a previous paper [5] it was shown that classical particles do entangle but with no observable evidence because all measurements a classical observer can conduct, breaks this coherence to provide the well-known distinguishable states. This study proposes that the main evidence for that primary entanglement is Newton's third law.

The concept of quantum entanglement started with the famous E.P.R Gedanken experiment [6] that was initially presented to demonstrate the incompleteness of quantum theory. Later, by D. Bohm's interpretation [7], the EPR state was introduced as two 1/2-spins particles that are entangled to form a zero-spin entity. The key point is that no matter their distance apart, the particles still belong to the same entity-the zero-spin particle. This characteristic is the source for an almost ideal encryption theory: If a code is carried by a pair of entangled particles, a local observer that observes only a single particle cannot either encrypt or duplicate the code because of the lack of information after interacting with the particle (for example an implication, see ref. [8]). Following ref. [5] for classical entanglement, if in some space, prior to the classical observer measurement, classical particles were entangled, it would be interesting to be able to determine the identifying feature that defines them as one. This paper does not answer that question but motivates us to dig deeper into the entangled side of classical mechanics with the hope that this complementary feature will be revealed in the future.

Introducing entanglement in classical mechanics requires the exploration of the relation between classical and quantum theories. In this paper, this variation is introduced through the concept of particle-labeling. In classical mechanics, labeling relates to the distinguishability and locality of classical particles. In our approach, however, labeling implies a simple quantum technique where a spanning labeling set is the basis to which all operators are projected to. Other approaches concerning locality are reviewed as follows:

Non-locality and entanglement were shown to be related topics [9]. In recent years, non-local theories of 
gravity and strings [10-23] have been presented. The attempts have been made through other theories to describe non-locality with quark confinement and anomalies [24,25]. Many theories introduce non-locality through the quantization of a class of relativistic non-local field equations, characterized by a non-local kinetic term in the Lagrangian [26]. El-Nabulsi [27] analyzes the implications of the quantum acceleratum operator, which is one of the main consequences of quantum non-local-in-time kinetic energy. Correspondingly, a non-local Heisenberg uncertainty is introduced.

As mentioned earlier, an attempt to find a relation between quantum and classical mechanics was performed by introducing entanglement into the local classical mechanics [5]. However, the locality was not introduced implicitly. Instead, it was assumed that the localized particles can be labeled, implying that in quantum terminology they are represented with a labeling basis of states. The main idea was that even classical particles can be entangled but the local nature of the classical observer that labels the particles, breaks this coherence to provide the local classical mechanics laws. Although the quantum terminology was implemented in the paper of ref [5], the main formalism was fundamental in the sense that operators such as force, were introduced fundamentally through a series of projecting operators with no quantum justification. In addition, Newton's second law was assumed to be fulfilled in its operator form. In this paper, we reformulate classical mechanics beginning with pure quantum principles. We then prove that the consistency of our theory must be accompanied with classical entanglement.

\section{Materials and Methods}

This is a pure mathematical work. We implemented techniques of Hilbert and mostly Folk spaces to introduce classical mechanics in a quantum-like terminology. No experiment or computer proceeding were implemented.

Funding: This research received no external funding.

Conflicts of Interest: The authors declare no conflict of interest.

\section{Introductory Section: Commutators Pictures and Representations}

A trivial identity is

$$
[\mathcal{H}, \overrightarrow{\mathcal{P}}]=-[\overrightarrow{\mathcal{P}}, \mathcal{H}]
$$

where in the Heisenberg picture we have

$$
\left[\mathcal{H}, \overrightarrow{\mathcal{P}}_{\langle E\rangle}\right]=i \hbar \frac{d \overrightarrow{\mathcal{P}}_{\langle E\rangle}}{d t}-i \hbar \frac{d}{d t} \overrightarrow{\mathcal{P}}
$$

with,

$$
\begin{aligned}
& \overrightarrow{\mathcal{P}}_{\langle E\rangle}=\mathcal{U}_{\langle E\rangle} \overrightarrow{\mathcal{P}} \mathcal{U}_{\langle E\rangle}^{\dagger} \\
& \mathcal{U}_{\langle E\rangle}=\exp \left[-i \frac{\mathcal{H} t}{\hbar}\right]
\end{aligned}
$$

We implement a conventional momentum operator, that is, $\overrightarrow{\mathcal{P}} \neq \overrightarrow{\mathcal{P}}(t)$ which causes the last term of eq. 2 to be canceled. This yield:

$$
\left[\mathcal{H}, \overrightarrow{\mathcal{P}}_{\langle E\rangle}\right]=i \hbar \frac{d \overrightarrow{\mathcal{P}}_{\langle E\rangle}}{d t}
$$

Using the space representation $\overrightarrow{\mathcal{P}}=-i \hbar \vec{\nabla}$ it can also be shown that,

$$
\left[\overrightarrow{\mathcal{P}}, \mathcal{H}_{\langle p\rangle}\right]=-i \hbar \vec{\nabla} \mathcal{H}_{\langle p\rangle}
$$


with,

$$
\begin{aligned}
& \mathcal{H}_{\langle P\rangle}=\mathcal{U}_{\langle p\rangle} \mathcal{H} \mathcal{U}_{\langle p\rangle}^{+} \\
& \mathcal{U}_{\langle p\rangle}=\exp \left[i \frac{\overrightarrow{\mathcal{P}} \cdot \vec{r}}{\hbar}\right] .
\end{aligned}
$$

where we assumed that $\mathcal{H} \neq \mathcal{H}(t)$.

Thus, if we substitute eqs. 4 and 5 into eq. 1, we obtain the classical law $\vec{\nabla} \mathcal{H}=-\dot{\overrightarrow{\mathcal{P}}}$ as an output of a trivial mathematical identity. Clearly, this is wrong as both operators are different in picture and representation. For example, the momentum operator in eq. 4 is presented in the energy form while in eq. 5 it is shown in the conventional form. Nevertheless, this paper will show that despite their different pictures, if we project both commutators of eqs. 4 and 5 onto a common representation, both sides of eq. 1 will be equatable, again, to the classical laws of mechanics. In our paper we implement the labeling basis of states as the common representation. In addition, we show that the phases that are accompanied by the exponential operators $\mathcal{U}_{\langle E\rangle}$ and $\mathcal{U}_{\langle p\rangle}$ generate the system dynamics.

\section{III.1. A Classical Terminology of a Post Collapse Entanglement}

An observer terminology describes the observable representation. By selecting a basis of states, the observer defines the concepts to be used in describing its measurement results[28]. Because the operators $\overrightarrow{\mathcal{P}}_{\langle E\rangle}$ and $\mathcal{H}_{\langle p\rangle}$ in eqs. 4 and 5 are represented differently, it make no sense to equate them. Mathematically, this means that either variable can appear simultaneously at different sides of the same equation provided that they possess the same dimensions, or that physical operators must appear with the same representation. One method of resolving this scenario is by projecting operators to the same representation. In this paper we suggest that this common representation becomes the labeling basis of states that distinguishes between particles [5], provided that each particle is associated solely with a well-defined momentum, and we can consider a diagonal momentum operator under the labeling basis of states. On the other hand, forces analysis is different. Consider a two bodies problem: In expressing mechanical relations between forces and momentum, each particle is associated with its individual momentum. However, in dealing with forces with the desire to solve the equations of motion, the two bodies which share forces causes an integration between both sets of equations. In a way similar to the encryption implication mentioned previously, we can say that both particles are essential to reveal all the system information. However, this is merely a of mathematical statement: "If you need to solve the equations of motion, you have to consider both particles and not only one". Experimentally, at any moment, forces can be detected on each isolated particle. This is because these particles are already in a collapsed state, having adjusted to describe each isolated particle.

To define mutual forces, we consider that under the labeling basis, the force operator is non-diagonal. Thus, the off-diagonal terms will correlate with different labeled states. It will be shown that this non-diagonal representation gives rise to the pre-collapsed entangled states.

\section{Force Operator $\overrightarrow{\mathcal{F}}_{\langle p\rangle}$ expressed in an off-Diagonal Momentum States}

In ref. [5], we introduce the force state $|\vec{F}\rangle$ as an eigenstate of the force operator where the operator was defined through a general formalism of projecting operators. In this section we implement eq. 5 to obtain an explicit expression for the force operator.

We first use the identities:

$$
\begin{aligned}
& \mathcal{U}_{\langle p\rangle}=\sum_{\vec{p}} \exp \left[i \frac{\vec{p} \cdot r}{\hbar}\right]|p\rangle\langle p|, \\
& \mathcal{U}_{\langle p\rangle}^{\dagger}=\sum_{\vec{p}}|p\rangle\langle p| \exp \left[-i \frac{\vec{p} \cdot r}{\hbar}\right]
\end{aligned}
$$

to obtain:

$$
\mathcal{H}_{\langle p\rangle}=\sum_{\vec{p}, \vec{p}^{\prime}} \exp \left[-i \frac{(\vec{p}-\vec{p}) \cdot r}{\hbar}\right]\left\langle\vec{p}|\mathcal{H}| \vec{p}^{\prime}\right\rangle|\vec{p}\rangle\left\langle\vec{p}^{\prime}\right|
$$


Using the momentum operator, which is expressed though projecting operators,

$$
\overrightarrow{\mathcal{P}}=\sum_{\vec{p}} \vec{p}|\vec{p}\rangle\langle\vec{p}|
$$

we find that,

$$
\begin{aligned}
& \mathcal{P} \mathcal{H}_{\langle p\rangle}=\sum_{\vec{p}, \vec{p}^{\prime}, \vec{p}^{\prime \prime}} \exp \left[-i \frac{(\vec{p}-\vec{p}) \cdot r}{\hbar}\right] \vec{p}^{\prime \prime}\left\langle\vec{p}^{\prime \prime} \mid \vec{p}\right\rangle\left\langle\vec{p}|\mathcal{H}| \vec{p}^{\prime}\right\rangle\left|\vec{p}^{\prime \prime}\right\rangle\left\langle\vec{p}^{\prime}\right| \overbrace{\vec{p}}^{\left\langle\vec{p}^{\prime \prime} \mid \vec{p}\right\rangle=\delta_{\vec{p}, \vec{p}^{\prime \prime}}} \\
& \Rightarrow \mathcal{P} \mathcal{H}_{\langle p\rangle}=\sum_{\vec{p}, \vec{p}^{\prime}} \vec{p} \exp \left[i \frac{\left(\vec{p}-\vec{p}^{\prime}\right) \cdot r}{\hbar}\right]\left\langle\vec{p}|\mathcal{H}| \vec{p}^{\prime}\right\rangle|\vec{p}\rangle\left\langle\vec{p}^{\prime}\right| .
\end{aligned}
$$

Similarly, it can be shown that

$$
\mathcal{H}_{\langle p\rangle} \mathcal{P}=\sum_{\vec{p}, \vec{p}^{\prime}} \vec{p}^{\prime} \exp \left[i \frac{\left(\vec{p}-\vec{p}^{\prime}\right) \cdot r}{\hbar}\right]\left\langle\vec{p}|\mathcal{H}| \vec{p}^{\prime}\right\rangle|\vec{p}\rangle\left\langle\vec{p}^{\prime}\right|
$$

yielding,

$$
\left[\overrightarrow{\mathcal{P}}, \mathcal{H}_{\langle p\rangle}\right]=\sum_{\vec{p}, \vec{p}^{\prime}}\left(\vec{p}-\vec{p}^{\prime}\right) \exp \left[i \frac{\left(\vec{p}-\vec{p}^{\prime}\right) \cdot r}{\hbar}\right]\left\langle\vec{p}|\mathcal{H}| \vec{p}^{\prime}\right\rangle|\vec{p}\rangle\left\langle\vec{p}^{\prime}\right|
$$

Consider a Hamiltonian with the form $\mathcal{H}=\frac{\mathcal{p}^{2}}{2 m}+\mathbf{V}$. Note that the factor $\vec{p}-\vec{p}^{\prime}$ eliminates the diagonal term of the kinetic energy which gives,

$$
\left[\overrightarrow{\mathcal{P}}, \mathcal{H}_{\langle p\rangle}\right]=\sum_{\vec{p}, \vec{p}^{\prime}}\left(\vec{p}-\vec{p}^{\prime}\right) \exp \left[i \frac{\left(\vec{p}-\vec{p}^{\prime}\right) \cdot r}{\hbar}\right]\left\langle\vec{p}|\mathbf{V}| \vec{p}^{\prime}\right\rangle|\vec{p}\rangle\left\langle\vec{p}^{\prime}\right|
$$

Defining the potential energy variable (its meaning, not as an operator)

$$
V_{p, p^{\prime}}(\vec{r})=\exp \left[i \frac{\left(\vec{p}-\vec{p}^{\prime}\right) \cdot r}{\hbar}\right]\left\langle\vec{p}|\mathbf{V}| \vec{p}^{\prime}\right\rangle
$$

we obtain,

$$
\left[\overrightarrow{\mathcal{P}}, \mathcal{H}_{\langle p\rangle}\right]=-i \hbar \vec{\nabla} \sum_{\vec{p}, \vec{p}^{\prime}} V_{p, p^{\prime}}(\vec{r})|\vec{p}\rangle\left\langle\vec{p}^{\prime}\right|
$$

Using eq. 5 and by identifying $\left[\overrightarrow{\mathcal{P}}, \mathcal{H}_{\langle p\rangle}\right]$ in the classical equation $\vec{F}=-\nabla H$, we obtain the force operator,

$$
\overrightarrow{\mathcal{F}}_{\langle p\rangle}(\vec{r})=-\vec{\nabla} \sum_{\vec{p}, \vec{p}^{\prime}} V_{p, p^{\prime}}(\vec{r})|\vec{p}\rangle\left\langle\vec{p}^{\prime}\right|
$$

or,

$$
\left[\overrightarrow{\mathcal{P}}, \mathcal{H}_{\langle p\rangle}\right]=i \hbar \overrightarrow{\mathcal{F}}_{\langle p\rangle}(\vec{r})
$$

97 We notice the following:

- $\overrightarrow{\mathcal{F}}_{\langle p\rangle}$ is an off-diagonal operator. This means that when applied to a momentum state it changes from $\vec{p}^{\prime}$ to $\vec{p}$ as a force operator.

- The gradient operation that describes a physical law, is generated by the phases exp $\left[i \frac{\left(\vec{p}-\vec{p}^{\prime}\right) \cdot r}{\hbar}\right]$.

- The time variable $t$ is missing in eq. 17. It appears later when we present momentum in the energy representation as described in section VI. Further discussion is provided in section VII.

Note that if $\left\langle\vec{p}|\mathbf{V}| \vec{p}^{\prime}\right\rangle=\left\langle\vec{p}^{\prime}|\mathbf{V}| \vec{p}\right\rangle$ and is real, the force operator is Hermitian; meaning that in the diagonal representation the orthogonal force-eigenstates with real eigenvalues are well defined quantities. 


\section{Force Operator $\overrightarrow{\mathcal{F}}_{\langle F\rangle}$ expressed in the Diagonal Force States}

The purpose of this section is to show that the force operator can be represented with the standard tools of quantum mechanics. That is, as a series of projecting operators accompanied with eigenvalues. Assuming a Hermitian $\overrightarrow{\mathcal{F}}$, its eigenstates $|\vec{F}\rangle$ generate a complete orthogonal basis of states that can be represented through the identity operator:

$$
\mathcal{I}_{\langle F\rangle}=\sum_{\vec{F}}|\vec{F}\rangle\langle\vec{F}|
$$

Thus, using the relation

$$
\overrightarrow{\mathcal{F}}_{\langle F\rangle}=\mathcal{I}_{\langle F\rangle} \overrightarrow{\mathcal{F}}_{\langle p\rangle} \mathcal{I}_{\langle F\rangle}^{\dagger}
$$

we obtain,

$$
\overrightarrow{\mathcal{F}}_{\langle F\rangle}=-\sum_{\vec{p}, \vec{p}^{\prime}} \sum_{\vec{F}, \vec{F}^{\prime}}\left(\vec{\nabla} V_{p, p^{\prime}}(\vec{r})\right)\left\langle\vec{F}^{\prime} \mid \vec{p}\right\rangle\left\langle\vec{p}^{\prime} \mid \vec{F}\right\rangle\left|\vec{F}^{\prime}\right\rangle\langle\vec{F}|
$$

As the force operator is diagonal in this representation, we obtain the relations that identify the state $|\vec{F}\rangle$ :

$$
\begin{aligned}
& \sum_{\vec{p}, \vec{p}^{\prime}}\left(\vec{\nabla} V_{p, p^{\prime}}(\vec{r})\right)\left\langle\vec{F}^{\prime} \mid \vec{p}\right\rangle\left\langle\vec{p}^{\prime} \mid \vec{F}\right\rangle=\vec{F} \delta_{\vec{F}, \vec{F}^{\prime}} \\
& \vec{F}=-\vec{\nabla} \sum_{\vec{p}, \vec{p}^{\prime}} V_{p, p^{\prime}}(\vec{r}) .
\end{aligned}
$$

Eqs. 21 enables us to write the force operator as a series of projecting operators:

$$
\overrightarrow{\mathcal{F}}_{\langle F\rangle}=\sum_{\vec{F}} \vec{F}|\vec{F}\rangle\langle\vec{F}|
$$

Using the definition $\left[\mathcal{P}, \mathcal{H}_{\langle p\rangle}\right]_{\langle F\rangle} \stackrel{\text { def }}{=} \mathcal{I}_{\langle F\rangle}\left[\mathcal{P}, \mathcal{H}_{\langle p\rangle}\right] \mathcal{I}_{\langle F\rangle}^{+}$we obtain:

$$
\left[\mathcal{P}, \mathcal{H}_{\langle p\rangle}\right]_{\langle F\rangle}=i \hbar \sum_{\vec{F}} \vec{F}|\vec{F}\rangle\langle\vec{F}|
$$

\section{VI. $\dot{\overrightarrow{\mathcal{P}}}$ in an off-Diagonal Energy Representation}

The momentum operator, shown in the Heisenberg picture is:

$$
\overrightarrow{\mathcal{P}}_{\langle E\rangle}=\sum_{E, E^{\prime}} \exp \left[\frac{i\left(E-E^{\prime}\right) t}{\hbar}\right]\left\langle E|\overrightarrow{\mathcal{P}}| E^{\prime}\right\rangle|E\rangle\left\langle E^{\prime}\right|
$$

In a similar manner to the previous calculations it can be shown that:

$$
\left[\mathcal{H}, \overrightarrow{\mathcal{P}}_{\langle E\rangle}\right]=\sum_{E, E^{\prime}}\left(E-E^{\prime}\right) \exp \left[\frac{i\left(E-E^{\prime}\right) t}{\hbar}\right]\left\langle E|\overrightarrow{\mathcal{P}}| E^{\prime}\right\rangle|E\rangle\left\langle E^{\prime}\right|
$$

Or,

$$
\left[\mathcal{H}, \overrightarrow{\mathcal{P}}_{\langle E\rangle}\right]=-i \hbar \frac{d \mathcal{P}\langle E\rangle}{d t}
$$

Defining the parameter

$$
\vec{P}_{E, E^{\prime}}(t)=\exp \left[\frac{i\left(E-E^{\prime}\right) t}{\hbar}\right]\left\langle E|\overrightarrow{\mathcal{P}}| E^{\prime}\right\rangle
$$

we obtain that

$$
\left[\mathcal{H}, \overrightarrow{\mathcal{P}}_{\langle E\rangle}\right]=-i \hbar \frac{d}{d t} \sum_{E, E^{\prime}} \vec{P}_{E, E^{\prime}}(t)|E\rangle\left\langle E^{\prime}\right|
$$


In this representation $t$ appears explicitly in the momentum operator

\section{Representations and Terminology}

Let us observe the classical equation

$$
\dot{\vec{P}}=-\vec{\nabla} H
$$

On the right side of eq. 28, we represent the classical Hamiltonian as a function of coordinates and momentum where in energy conserving system, time is explicitly missing despite the fact that $\vec{r}$ and $\vec{p}$ depend on time. Time dependence appear explicitly on the left side of eq. 28 where the momentum is now expressed as a function of time. Following eqs. 17 and 27 we find the same terminology in eq. 17 where the system is represented through momentum and space only while time appears explicitly in the momentum description in eq. 27.

\section{Common Representation}

In order to utilize the trivial identity $[\mathcal{H}, \overrightarrow{\mathcal{P}}]=-[\overrightarrow{\mathcal{P}}, \mathcal{H}]$ in equations 17 and 27, we need to projects all operators onto the same basis of states. As in classical mechanics where bodies are distinguishable, we choose the common basis of states to be the labeling set, $|s\rangle, s=1,2, \ldots,[5]$ where the index $s$ labels the distinguishable bodies.

Defining the corresponding complete and orthogonal basis of states, $|s\rangle$, we can define the identity operators,

$$
\mathcal{I}=\sum_{s}|s\rangle\langle s|
$$

Substituting the relations $\mathcal{I} \overrightarrow{\mathcal{F}} \mathcal{I}^{\dagger}$ and $\mathcal{I} \overrightarrow{\mathcal{P}} \mathcal{I}^{\dagger}$, in eqs. 15 and 27 we obtain:

$$
\overrightarrow{\mathcal{F}}_{\langle s\rangle}=-\left[\overrightarrow{\mathcal{P}}, \mathcal{H}_{\langle p\rangle}\right]_{\langle s\rangle}=i \hbar \vec{\nabla} \sum_{s, s^{\prime}} \sum_{\vec{p}, \vec{p}^{\prime}} V_{p, p^{\prime}}(\vec{r})\langle s \mid \vec{p}\rangle\left\langle\vec{p}^{\prime} \mid s^{\prime}\right\rangle|s\rangle\left\langle s^{\prime}\right|
$$

and,

$$
\dot{\overrightarrow{\mathcal{P}}}_{\langle s\rangle}=\left[\mathcal{H}, \overrightarrow{\mathcal{P}}_{\langle E\rangle}\right]_{\langle s\rangle}=-i \hbar \frac{d}{d t} \sum_{s, s^{\prime}} \sum_{E, E^{\prime}} \vec{P}_{E, E^{\prime}}(t)\langle s \mid E\rangle\left\langle E^{\prime} \mid s^{\prime}\right\rangle|s\rangle\left\langle s^{\prime}\right| .
$$

Implementing eq. 1 for the common representation we obtain the operator equation:

$$
\overrightarrow{\mathcal{F}}_{\langle s\rangle}=\dot{\overrightarrow{\mathcal{P}}}_{\langle s\rangle}
$$

This equation that was considered as a postulate in ref. [5] is now derived from basic quantum principles.

\section{Diagonal Representation of Momentum in the Labeling Basis of States}

With regards to individual particles, classical mechanics allows particles to share forces (through mutual interactions) while each particle can still be associated with a distinguishable momentum. In quantum terminology, this means that the momentum operator is diagonal under the labeling basis of states. Assuming:

$$
\sum_{E, E^{\prime}} \vec{P}_{E, E^{\prime}}(t)\langle s \mid E\rangle\left\langle E^{\prime} \mid s^{\prime}\right\rangle=\vec{p}_{s}(t) \delta_{s, s^{\prime}}
$$

we obtain from eq. 31 the momentum operator's diagonal form:

$$
\overrightarrow{\mathcal{P}}_{\langle s\rangle}=\sum_{s} \vec{p}_{s}(t)|s\rangle\langle s|
$$


124

\section{Internal and External Forces}

For simplicity we define the following variable:

$$
\vec{F}_{s, s^{\prime}} \stackrel{\text { def }}{=}-\vec{\nabla} \sum_{\vec{p}, \vec{p}^{\prime}} V_{p, p^{\prime}}(\vec{r})\langle s \mid \vec{p}\rangle\left\langle\vec{p}^{\prime} \mid s^{\prime}\right\rangle
$$

to obtain from eq. 30 the force operator,

$$
\overrightarrow{\mathcal{F}}_{\langle s\rangle}=\sum_{s, s^{\prime}} \vec{F}_{s, s^{\prime}}|s\rangle\left\langle s^{\prime}\right| .
$$

To distinguish between solitary and mutual interaction forces, we separate $\overrightarrow{\mathcal{F}}_{\langle s\rangle}$ into the diagonal terms $\left|s^{\prime}\right\rangle\langle s|=\delta_{s, s^{\prime}}$ and the off-diagonal terms. This yields:

$$
\overrightarrow{\mathcal{F}}_{\langle s\rangle}=\sum_{s} \overrightarrow{\mathbf{F}}_{s}
$$

where $\overrightarrow{\mathbf{F}}_{s}$, the net force operator that acts upon a s-particle is:

$$
\overrightarrow{\mathbf{F}}_{s}=\vec{F}_{s}|s\rangle\left\langle s\left|+\sum_{s^{\prime}} \vec{F}_{s \neq s}\right| s^{\prime}\right\rangle\langle s| .
$$

\section{Interactions, Newton Third Law and Hermiticy}

With the purpose of reconstructing Newton's third law, we obtain the relation

$$
\vec{F}_{s^{\prime} \neq s}=-\vec{F}_{s \neq s^{\prime}} \text { Note that on both sides, } s \text { and } s^{\prime} \text { were switched. }
$$

Defining the interaction force magnitude as $\left|\vec{F}_{s, s^{\prime}}\right|=\left|\vec{F}_{s^{\prime} \neq s}\right|$ we obtain:

$$
\overrightarrow{\mathbf{F}}_{\mathcal{S}}=\vec{F}_{S}|s\rangle\left\langle s\left|+\sum_{s^{\prime}}\right| \vec{F}_{s^{\prime}, s}\right|\left(\left|s^{\prime}\right\rangle\langle s|-| s\rangle\left\langle s^{\prime}\right|\right)
$$

Following the convention of an Hermit observable and noticing that $\overrightarrow{\mathbf{F}}_{S}$ of eq. 40 is not such, we suggest the replacement, $\left|\vec{F}_{s^{\prime}, s}\right|\left(\left|s^{\prime}\right\rangle\langle s|-| s\rangle\left\langle s^{\prime}\right|\right) \rightarrow i\left|\vec{F}_{s^{\prime}, s}\right|\left(\left|s^{\prime}\right\rangle\langle s|-| s\rangle\left\langle s^{\prime}\right|\right)$ to obtain the Hermit operator:

$$
\overrightarrow{\mathbf{F}}_{s}=\vec{F}_{s}|s\rangle\left\langle s\left|+i \sum_{s^{\prime}}\right| \vec{F}_{s^{\prime}, s}\right|\left(\left|s^{\prime}\right\rangle\langle s|-| s\rangle\left\langle s^{\prime}\right|\right)
$$

$129 \quad$ Note that $\sum_{s} \overrightarrow{\mathbf{F}}_{S}=\sum_{S} \vec{F}_{S}|s\rangle\langle s|$.

130 XII. Second Quantization

Classical mechanics includes particles interactions and is therefore useful to introduce our system with multi-particle algebra. Now the states belong to the Fock space.

Following ref. [5] we use the transformation

$$
\begin{aligned}
& |s\rangle\langle s| \rightarrow a_{s}^{\dagger} a_{s} \\
& \left|s^{\prime}\right\rangle\langle s| \rightarrow a_{s^{\prime}}^{+} a_{s} \\
& |s\rangle\left\langle s^{\prime}\right| \rightarrow a_{s}^{+} a_{s^{\prime}},
\end{aligned}
$$


where $a$ and $a^{\dagger}$ behave like spin $\frac{1}{2}$ fermionic annihilation and creation operators,

$$
\begin{aligned}
& a_{s}|0\rangle_{s^{\prime}}=0, \quad a_{s}^{\dagger}|0\rangle_{s^{\prime}}=|1\rangle_{s} \delta_{s s^{\prime}} \\
& a_{s}|1\rangle_{s^{\prime}}=|0\rangle_{s} \delta_{s s^{\prime}}, \quad a_{s}^{\dagger}|1\rangle_{s^{\prime}}=0 .
\end{aligned}
$$

The momentum operator becomes:

$$
\overrightarrow{\mathcal{P}}_{\langle s\rangle}=\sum_{s} \vec{p}_{s}(t) a_{s}^{\dagger} a_{s}
$$

and Eq. 41 is now modified to,

$$
\overrightarrow{\mathbf{F}}_{S}=\vec{F}_{S} a_{s}^{\dagger} a_{s}+i \sum_{s^{\prime}}\left|\vec{F}_{s^{\prime}, s}\right|\left(a_{s^{\prime}}^{\dagger} a_{s}-a_{s}^{\dagger} a_{s^{\prime}}\right)
$$

whereas, the total force operator was:

$$
\overrightarrow{\mathcal{F}}_{\langle s\rangle}=\sum_{s} \overrightarrow{\mathbf{F}}_{S}
$$

Vacuum state The state in which no particle is observed:

$$
|\varnothing\rangle_{\langle s\rangle}=\prod_{s=1}|0\rangle_{s}
$$

For that state we obtain

$$
\overrightarrow{\mathcal{F}}_{\langle s\rangle}|\varnothing\rangle=0
$$

Close system state The state in which all s-states are occupied with a single fermionic particle:

$$
|C . S .\rangle=\prod_{s=1}|1\rangle_{s}
$$

Inserting $\mid$ C.S. $\rangle$ into Eqs. 45 and 46, and noticing that the asymmetric term is canceled, we obtain

$$
\left.\left.\overrightarrow{\mathcal{F}}_{S} \mid \text { C.S. }\right\rangle=\left(\sum_{s} \vec{F}_{S}\right) \mid \text { C.S. }\right\rangle .
$$

Implementing eqs. 44 and 32, we obtain the known classical relation

$$
\sum_{s} \vec{F}_{s}=\dot{\vec{P}}_{\text {total }}
$$

${ }_{134} \quad$ where $\vec{P}_{\text {total }} \stackrel{\text { def }}{=} \sum_{s} \vec{P}_{s}$.

135

\section{XII.2. Single Particle States}

In a single particle state, only one state (s) is occupied while all others are empty:

$$
|s\rangle=|1\rangle_{s} \prod_{s^{\prime} \neq s}|0\rangle_{s^{\prime}}
$$

Substituting in eq. 46 we obtain,

$$
\overrightarrow{\mathcal{F}}_{\langle s\rangle}|s\rangle=\vec{F}_{s}|s\rangle+i \sum_{s^{\prime}}\left|\vec{F}_{s, s^{\prime}}\right|\left|s^{\prime}\right\rangle .
$$


Considering that $\forall s^{\prime} \neq s,\left\langle s \mid s^{\prime}\right\rangle=0$, we obtain that $\forall s^{\prime}, \vec{F}_{s^{\prime} \rightarrow s}=0$. This means that the single particle approach cannot represent internal forces and consequently Newton's third law. In section XIII, we resolve this difficulty by introducing entangled states.

\section{Entanglement}

We implement single particle states of the particles pair $s_{1}$ and $s_{2}$ to generate doubles of entangled states:

$$
\text { For } s_{1} \neq s_{2},\left\{\begin{array}{l}
\left|\psi_{+}\right\rangle=\left|s_{1}\right\rangle+i\left|s_{2}\right\rangle \\
\left|\psi_{-}\right\rangle=\left|s_{2}\right\rangle-i\left|s_{1}\right\rangle
\end{array}, \quad\left|\left\langle\psi_{+} \mid \psi_{+}\right\rangle\right|^{2}=\left|\left\langle\psi_{-} \mid \psi_{-}\right\rangle\right|^{2}=2,\right.
$$

with the single particle states:

$$
\begin{aligned}
& \left|s_{1}\right\rangle=|1\rangle_{s_{1}} \prod_{s \neq s_{1}}|0\rangle_{s} \quad \begin{array}{c}
\text { The observer detects only } \\
\text { the particle which is labeled as } s_{1}
\end{array} \\
& \left|s_{2}\right\rangle=|1\rangle_{s_{2}} \prod_{s \neq s_{2}}|0\rangle_{s} \quad \begin{array}{c}
\text { The observer detects only } \\
\text { the particle which is labeled as } s_{2}
\end{array}
\end{aligned}
$$

According to eq. 45 , each $s_{1}$ or $s_{2}$ particle is associated with a force operators $\overrightarrow{\mathbf{F}}_{s_{1}}$ or $\overrightarrow{\mathbf{F}}_{s_{2}}$, as follows:

$$
\begin{aligned}
& \overrightarrow{\mathbf{F}}_{s_{1}}=\vec{F}_{s_{1}} a_{s_{1}}^{\dagger} a_{s_{1}}+i \sum_{s}\left|\vec{F}_{s_{s} s_{1}}\right|\left(a_{s}^{\dagger} a_{s_{1}}-a_{s_{1}}^{\dagger} a_{s}\right) \quad \text { The fon parce acting } \\
& \overrightarrow{\mathbf{F}}_{s_{2}}=\vec{F}_{s_{2}} a_{s_{2}}^{\dagger} a_{s_{2}}+i \sum_{s}\left|\vec{F}_{s_{1} s_{2}}\right|\left(a_{s}^{\dagger} a_{s_{2}}-a_{s_{2}}^{\dagger} a_{s}\right) \quad \text { The forceacting }
\end{aligned}
$$

where we replaced the dummy index $s^{\prime}$ with $s$.

We then obtain:

$$
\overrightarrow{\mathbf{F}}_{s_{1}}\left|\psi_{+}\right\rangle=\vec{F}_{s_{1}}|1\rangle_{s_{1}} \prod_{s \rightarrow s_{1}}|0\rangle_{s}+i \sum_{\sigma}\left|\vec{F}_{\sigma, s_{1}}\right||1\rangle_{\sigma} \prod_{s \neq \sigma}|0\rangle_{s}
$$

and in eq. 55's symbol terminology,

$$
\overrightarrow{\mathbf{F}}_{s_{1}}\left|\psi_{+}\right\rangle=\vec{F}_{s_{1}}\left|s_{1}\right\rangle+i \sum_{\sigma}\left|\vec{F}_{\sigma, s_{1}}\right|\left|s_{\sigma}\right\rangle
$$

and in the same manner we obtain:

$$
\begin{aligned}
\overrightarrow{\mathbf{F}}_{s_{2}}\left|\psi_{-}\right\rangle & =\vec{F}_{s_{2}}\left|s_{2}\right\rangle+i \sum_{\sigma}\left|\vec{F}_{\sigma, s_{2}}\right|\left|s_{\sigma}\right\rangle \\
\overrightarrow{\mathbf{F}}_{s_{1}}\left|\psi_{-}\right\rangle & =-i \vec{F}_{s_{1}}\left|s_{1}\right\rangle+\sum_{\sigma}\left|\vec{F}_{\sigma, s_{1}}\right|\left|s_{\sigma}\right\rangle \\
\overrightarrow{\mathbf{F}}_{s_{2}}\left|\psi_{+}\right\rangle & =i \vec{F}_{s_{2}}\left|s_{2}\right\rangle-\sum_{\sigma}\left|\vec{F}_{\sigma, s_{2}}\right|\left|s_{\sigma}\right\rangle
\end{aligned}
$$

To retrieve classical results, we consider an observer that by performing measurements, collapses the entanglement into the distinguishable (labeled) particles. Consequently, they detect only average quantities. Implementing eqs. 54, 58 and 59 we obtain:

$$
\begin{aligned}
& \begin{array}{l}
\text { The net force } \\
\text { acting on particle 1: }
\end{array} \Rightarrow\left\{\begin{array}{l}
\left\langle\psi_{+}\left|\overrightarrow{\mathbf{F}}_{s_{1}}\right| \psi_{+}\right\rangle=\vec{F}_{s_{1}}+\mid \vec{F}_{s_{1}, s_{2}} \\
\left\langle\psi_{-}\left|\overrightarrow{\mathbf{F}}_{s_{1}}\right| \psi_{-}\right\rangle=\vec{F}_{s_{1}}+\left|\vec{F}_{s_{1}, s_{2}}\right|
\end{array}\right. \\
& \begin{array}{c}
\text { The net force } \\
\text { acting on particle 2: }
\end{array} \Rightarrow\left\{\begin{array}{l}
\left\langle\psi_{-}\left|\overrightarrow{\mathbf{F}}_{s_{2}}\right| \psi_{-}\right\rangle=\vec{F}_{s_{2}}-\left|\vec{F}_{s_{1}, s_{2}}\right| \\
\left\langle\psi_{+}\left|\overrightarrow{\mathbf{F}}_{s_{2}}\right| \psi_{+}\right\rangle=\vec{F}_{s_{2}}-\left|\vec{F}_{s_{1}, s_{2}}\right|
\end{array}\right.
\end{aligned}
$$


Comparing the terms $\left\langle\psi_{+}\left|\overrightarrow{\mathbf{F}}_{s_{1}}\right| \psi_{+}\right\rangle$and $\left\langle\psi_{+}\left|\overrightarrow{\mathbf{F}}_{s_{2}}\right| \psi_{+}\right\rangle$or $\left\langle\psi_{-}\left|\overrightarrow{\mathbf{F}}_{s_{1}}\right| \psi_{-}\right\rangle$and $\left\langle\psi_{-}\left|\overrightarrow{\mathbf{F}}_{s_{2}}\right| \psi_{-}\right\rangle$we find that Newton's third law has been reformulated. The third law is what is left from a primary entanglement of classical particles.

\section{Classical Observer}

In the previous section we associated measurement outputs with averages. This means that the so-called single observation in fact includes a multitude of measurements forming an ensemble that allows the detection of average quantities.

\section{Results}

In this paper, we reformulated classical mechanics laws from pure quantum principles. We showed that the identity $[\mathcal{H}, \overrightarrow{\mathcal{P}}]=-[\overrightarrow{\mathcal{P}}, \mathcal{H}]$ can be implemented to reformulate the laws of classical mechanics provided that the following two-step procedure is to be followed: First, the right side operator of a commutator is to be pictured with the corresponding unitary operators of the left side operator. For example, right side operator $\overrightarrow{\mathcal{P}}_{\langle E\rangle}$ in $\left[\mathcal{H}, \overrightarrow{\mathcal{P}}_{\langle E\rangle}\right]$ is represented by the left side states and phases of the Hamiltonian. The second step is to project both commutators (such as in eqs. 4 and 5) onto a common basis. Then, can we equate both commutators to obtain a physical law. We suggest that by following this procedure and by implementing other operators, it may be possible to extend this approach to find or reconstruct other physical laws.

As shown earlier, using this approach to reconstruct classical mechanics laws, Newton's third law corresponds with entanglement. That is to say, prior to a classical mechanical measurement, the labeling states were defined as quantum states that cannot be factored as a product of the states of its local constituents. We showed that the remaining evidence for this coherence is the internal forces. Philosophically speaking, we realize that beyond classical mechanics, there is another reality in which even classical particles are entangled. As the classical observer can measure only a limited basis of states, entangled states become impossible to measure.

\section{References}

1. Author1, T. The title of the cited article. Journal Abbreviation 2008, 10, 142-149.

2. Author2, L. The title of the cited contribution. In The Book Title; Editor1, F., Editor2, A., Eds.; Publishing House: City, Country, 2007; pp. 32-58.

Sample Availability: Samples of the compounds ...... are available from the authors.

\section{References}

3. F. Nicolai Friis, G.Vitagliano, M. Malik and M. Huber, arXiv.org > quant-ph > arXiv:1906.10929 (2019)

4. E. Chitambar and Gi. Gour, Rev. Mod. Phys. 91, (2019)

5. Y. Roth, Results in Physics, 14 (2019).

6. A. Einstein, B. Podolsky, and N. Rosen, Phys. Rev., 47 (1935).

7. Hiley B.J. (2009) Bohm's Approach to the EPR Paradox. In: Greenberger D., Hentschel K., Weinert F. (eds) Compendium of Quantum Physics. Springer, Berlin, Heidelberg

8. C. H. Bennett, G. Brassard, Theoretical Computer Science, 560,1 (2014)

9. F. Buscemi, Phys. Rev. Lett., 108 (2012).

10. E.T. Tomboulis, Phys. Rev D, 92, 125037 (2015)

11. E.T. Tomboulis, arXiv:hep-th/9702146.

12. L. Modesto, Phys. Rev. D 86, 044005 (2012).

13. T. Biswas, E. Gerwick, T. Koivisto, and A. Mazumdar, Phys. Rev. Lett. 108, 031101 (2012).

14. T. Biswas, A. Conroy, A.S. Koshelev, and A. Mazumdar, Classical Quantum Gravity 31,015022(2014).

15. S.Talaganis, T. Biswas, and A. Mazumdar, Classical Quantum Gravity 32, 215017 (2015).

16. L. Modesto, Astron. Rev. 8, 4 (2013); arXiv:1402.6795. 
185 17. F. Briscese, L. Modesto, and S.Tsujikawa, Phys. Rev. D 89, 024029 (2014).

186 18. G. Calcagni and L. Modesto, Phys. Rev. D, 92, 125037 (2015); 125037-17 91, 124059 (12015).

187 19. L. Modesto and L. Rachwal, Nucl. Phys. B889, 228 (2014).

188 20. 8] M. Isi, J. Mureika, and P. Nocolini, J. High Energy Phys. 11 (2013) 139.

189 21. V.P. Frolov, Phys. Rev. Lett. 115, 051102 (2015); V.P. Frolov, A. Zelnikov, and T. de Paula Netto, J. High Energy 190 Phys. 06, 107 (2015).

191 22. V.P. Frolov and A. Zelnikov, arXiv:1509.03336.

192 23. N.Barnabyand N.Kamran, J.High Energy Phys. 2, 008 (2008).

193 24. M. K. Volkov and G. V. Efimov, Sov. Phys. Usp. 23, 94 (1980).

194 25. N. V. Krasnikov, Theor. Math. Phys. 73, 1184 (1987).

195 26. D. G. Barci, L. E. Oxman, M. Rocca, Int. J. Mod. Phys., A11, 2111-2126 (1996).

27. Rami Ahmad El-Nabulsi, Quantum Stud.: Math. Found. https://doi.org/10.1007/s40509-018-0170-1.

198 28. Y. Roth J. Phys. Commun., 3045002 (2019)

199

29. N. Friis, G. Vitagliano, M. and M. Huber, Nature Reviews Physics, 1, pages72-87 (2019)

200 